\title{
Healthy Hip Joints Have Different Macroscopic and Microscopic Capsular Nerve Architecture Compared With Hips With Osteoarthritis, Femoroacetabular Impingement Syndrome, and Developmental Dysplasia of the Hip: A Systematic Review
}

\author{
Carlos J. Meheux, M.D., Takashi Hirase, M.D., David Dong, B.S., Terry A. Clyburn, M.D., \\ and Joshua D. Harris, M.D.
}

\begin{abstract}
Purpose: To perform a systematic review to identify macroscopic and microscopic patterns and differences in hip capsule innervation between normal hips and hips with osteoarthritis (OA), femoroacetabular impingement (FAI) syndrome, and developmental dysplasia of the hip (DDH). Methods: A systematic review was performed using Preferred Reporting Items for Systematic reviews and Meta-Analyses guidelines. Multiple databases were searched for both clinical and basic science laboratory studies on hip capsule innervation. Non-innervation capsule and non-human animal studies were excluded. Macroscopic and microscopic differences in capsular innervation between normal hips, and hips with OA, FAI, and DDH were analyzed. Methodological quality assessment of all studies included in this review was completed using the Methodological Index for Non-randomized Studies. Results: Ten articles were analyzed (263 specimens; 211 patients, 52 cadavers; mean Methodological Index for Non-randomized Studies 10/16). The hip capsule is innervated by the sciatic and superior gluteal nerves posterosuperiorly, nerve to quadratus femoris and inferior gluteal nerve posteroinferiorly, and femoral and obturator nerves anteriorly. The anterior-superior capsule between 1:00 and 2:30 o'clock on a right hip is a safe internervous zone. The superolateral capsule has the greatest density of mechanoreceptors and sensory fibers. OA is associated with a greater expression of nerve fibers compared with normal hips but does not correlate with pain or disability. No significant differences were found in nerve fiber expression among patients with DDH, FAI, or normal hips. A negative correlation is seen with aging and pain fiber expression. Conclusions: The hip capsule has a complex macroscopic and microscopic innervation pattern with varying nerve fiber expression from at least 6 separate peripheral nerves. OA is associated with a greater expression of nerve fibers, although nerve fiber expression does not correlate with painful pathology. Level of Evidence: IV, Systematic review of level I-IV studies.
\end{abstract}

$\mathbf{P}$ ain is an essential symptom of hip osteoarthritis (OA), femoroacetabular impingement syndrome (FAI), and developmental dysplasia of the hip (DDH). ${ }^{1-3}$ Hip pain may be severe, equated to a pain "worse than

From Houston Methodist Orthopedic and Sports Medicine, Houston, Texas, U.S.A.

The authors report the following potential conflicts of interest or sources of funding:

T.A.C. reports AAOS: board or committee member; Conformis: research support; stock or stock options; Nimbic Systems: IP royalties. J.D.H. reports AAOS: board or committee member; American Orthopaedic Society for Sports Medicine: board or committee member; Arthroscopy: editorial or governing board; Arthroscopy Association of North America: board or committee member; Arthrex/Medinc of Texas: research support; DePuy, A Johnson $\theta$ Johnson Company: research support; Frontiers In Surgery: editorial or governing board; NIA Magellan: paid consultant; SLACK Incorporated: publishing royalties, financial or material support; Smith $\theta$ Nephew: paid presenter or death." ${ }^{\prime 4}$ Nociception (peripheral noxious tissue injury detected by nociceptors) and pain (higher level central perception and interpretation of that peripheral tissue damage) are not the same entity, as nociception can

speaker, paid consultant, research support; Ossur: paid speaker. Full ICMJE author disclosure forms are available for this article online, as supplementary material.

Received May 11, 2020; accepted August 10, 2020.

Address correspondence to Joshua D. Harris, M.D., Houston Methodist Orthopedic and Sports Medicine, 6445 Main St., Suite 2500, Houston, TX 77030.E-mail: joshuaharrismd@gmail.com

(C) 2020 THE AUTHORS. Published by Elsevier Inc. on behalf of the Arthroscopy Association of North America. This is an open access article under the CC BY-NC-ND license (http://creativecommons.org/licenses/by-nc-nd/4.0/). 2666-061X/20701

https://doi.org/10.1016/j.asmr.2020.08.015 
occur without pain. This is an important distinction in the hip, considering the high prevalence of asymptomatic imaging abnormalities. ${ }^{5}$ Because of these complexities within pain and nociception, pain may be a singular indication for surgical intervention in patients with hip pathology. Therefore, the most important aim of treatment is pain relief and improvement of physical function and overall quality of life.

Hip pain results from the stimulation of free endings of nociceptors of slow-conducting (unmyelinated axons, C-fibers) and faster conducting (thinly myelinated axons, A-delta fibers) nerve fibers. The perception of pain in the hip may be transmitted by autonomic nerve supply of the intraosseous blood vessels, innervation of the synovium and capsule, and periarticular muscles. ${ }^{6,7}$ This effect can be amplified by biochemical agents, such as bradykinin or histamine, which may activate the endings of nerve fibers, triggering pain, whereas other agents, such as substance $\mathrm{P}$ or prostaglandins, mainly enhance the sensitivity to applied stimuli. ${ }^{8,9}$ Moreover, a persistent noxious, mechanical, or thermal stimulation input can be involved in developing or increasing central sensitization and release processes, leading to or supporting chronic pain. ${ }^{10}$

The hip capsule is a complex anatomical structure that plays an important role in native hip stability. ${ }^{11}$ The capsule is a condensation of the iliofemoral, pubofemoral, and ischiofemoral ligaments and the zona orbicularis. While capsular management in hip-preservation surgery has evolved significantly over the recent past, the main interest lies in its contribution to joint stability. Although the issue is not "solved," capsule preservation with repair is more of a rule now, rather than an exception. ${ }^{12,13}$ Unfortunately, the controversial issues have not investigated the capsule's innate role in pain generation. The presence and quantity of neuronal elements in the joint capsule may implicate the capsule itself in the pathogenesis of preor post-treatment pain-too loose, too tight, too irregular, too thick, too thin, too much adhesion formation (capsulolabral adhesions), or foreign body irritation (suture). A thorough understanding of the normal and diseased hip joint capsule neuroanatomy can help guide treatment, especially with surgical intervention that involves capsulotomy, capsulectomy, capsular repair, or capsular plication. The purpose of this study was to perform a systematic review to identify macroscopic and microscopic patterns and differences in hip capsule innervation between normal hips and hips with OA, FAI syndrome, and DDH. It was hypothesized that normal hips would have different macroscopic and microscopic capsular nerve architecture compared with hips with OA, FAI and DDH.

\section{Methods}

\section{Design and Search Strategy}

A systematic review was registered on PROSPERO on April 18, 2018 (registration ID: CRD 42018094248).
PRISMA (Preferred Reporting items for Systematic reviews and Meta-Analyses) guidelines were followed. ${ }^{14}$ Under the PROSPERO registration, similar previous systematic reviews and meta-analyses were sought, and none were identified. Separate electronic searches of the following databases were conducted: MEDLINE, Cochrane Central Register of Controlled Trials, SCOPUS and Sport Discus. The searches were performed on April 26, 2020. To ensure a stringent search strategy of relevant literature, key words including "hip capsule," "innervation," "hip osteoarthritis," "femoroacetabular impingement," and "developmental dysplasia" were combined with Boolean operators to develop a search protocol (Appendix Table 1). A hand search of the included reference lists also was performed to further minimize unintentional exclusion of relevant studies.

\section{Eligibility Criteria}

Levels I, II, III, and IV evidence (based on Oxford Centre for Evidence Based Medicine) clinical outcome therapeutic, diagnostic, or prognostic studies and basic science laboratory studies (including human cadaver specimens) published in English language that involved normal or diseased human hip capsular innervation were eligible for inclusion. ${ }^{15}$ Differences in macroscopic and microscopic differences in innervation of the normal hip and hips with OA, FAI, and DDH were sought, extracted, and analyzed. Macroscopic innervation studies were defined as any investigation of nerves visible with the naked eye without microscopic amplification. Microscopic innervation studies were defined as any investigation reporting outcomes of microscopic analysis of innervation of the hip capsule. Hips with arthritis were defined in a variety of ways: the study explicitly reported "osteoarthritis," joint space width less than $2 \mathrm{~mm}$, Tönnis grade 2 or 3 , or the presence of osteophytes, subchondral sclerosis, and subchondral cysts. Hips with FAI were defined based on patient symptoms, clinical signs on physical examination, and radiographic features consistent with either cam (alpha angle greater than $55^{\circ}$ on any view, 3-dimensional head-neck junction asphericity) or pincer (lateral center edge angle greater than $40^{\circ}$, anterior center edge angle greater than $40^{\circ}$, protrusio acetabulae, posterior wall and ischial spine signs [acetabular retroversion], or crossover sign with computed tomography evidence of focal loss of cranial acetabular anteversion) morphology. Hips with DDH were defined based on patient symptoms, clinical signs of physical examination, and radiographic features consistent with dysplasia (broken Shenton's line, lateral center edge angle less than 15 to $20^{\circ}$, anterior center edge angle less than 15 to $20^{\circ}$, Tönnis angle greater than 10 to $15^{\circ}$, femoral head extrusion index greater than $25 \%$, or computed tomography evidence of anterior, lateral, posterior, or global femoral head uncoverage). Non-human animal model, level $\mathrm{V}$ evidence, studies that did not focus on hip capsular innervation, and non-English 
Fig 1. PRISMA flowchart showing application of selection criteria to the studies identified with the search strategy. (PRISMA, Preferred Reporting items for Systematic reviews and Meta-Analyses.)
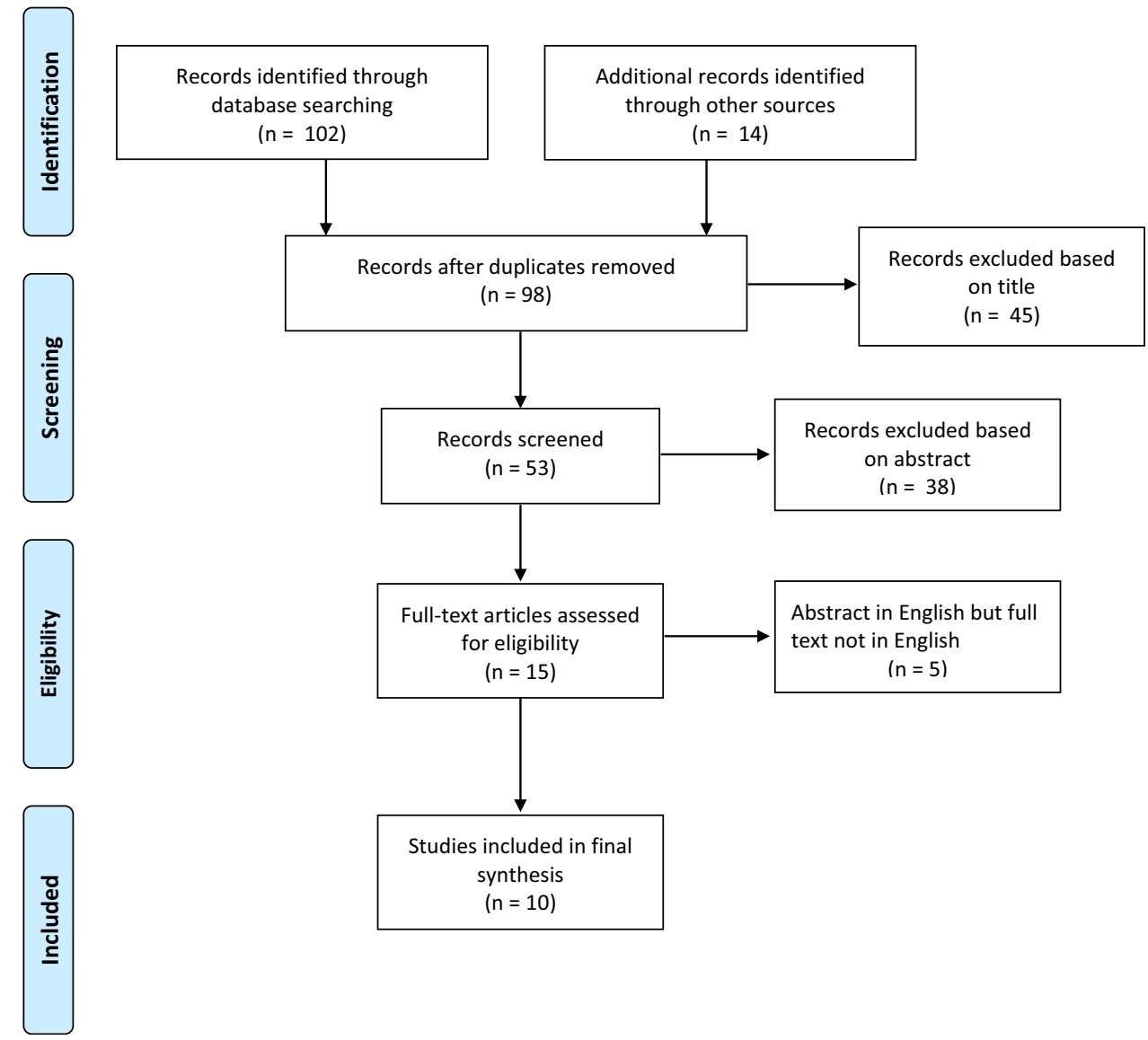

Additional records identified through other sources $(n=14)$

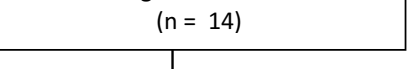

Studies included in fina synthesis

Methodological Index for Non-randomized Studies was $10.0 \pm 3.5$. results were reviewed for duplicates and the inclusion criteria to determine articles that were included in the final analysis (Fig 1).

\section{Data Extraction and Analysis}

Two of the authors independently reviewed, and data were extracted from all articles using methodology recommended by Harris et al. ${ }^{16}$ The study type, design, methods, and level of evidence were identified. Methodological quality assessment of all studies included in this review was completed using the Methodological Index for Non-randomized Studies. ${ }^{17}$ For clinical studies, the levels of evidence were assigned based on the Oxford Centre for Evidence-Based Medicine. ${ }^{15}$ Because of the heterogeneity of outcome measures and low level of evidence, a best-evidence synthesis was used instead of a meta-analysis. ${ }^{18}$

\section{Results}

Ten studies were included in the systematic review (Fig 1). ${ }^{19-28}$ There were 4 cadaveric and 6 in vivo studies describing 263 specimens (Table 1). Mean

\section{Macroscopic Innervation of the Hip Capsule}

Three cadaveric studies describing 44 cadavers investigated macroscopic innervation of normal hip capsules to find that the hip capsule is innervated by the sciatic and superior gluteal nerves posterosuperiorly (ischiofemoral ligament), nerve to quadratus femoris and inferior gluteal nerves posteroinferiorly (ischiofemoral ligament), and femoral and obturator nerves anteriorly (iliofemoral and pubofemoral ligament) (Fig 2). 20,22,28 One study demonstrated that the $45^{\circ}$ arch in the anterosuperior right hip capsule between 1:00 and 2:30 o'clock is a safe internervous zone for capsular incision during surgery. ${ }^{22}$

\section{Microscopic Innervation of the Hip Capsule}

One cadaveric and 6 in vivo studies (8 cadavers, 211 live humans) performed microscopic analyses of hip capsule innervation. ${ }^{19,21,23-26}$ One study used gold chloride staining in 8 cadavers without a known hip pathology to map nerve fiber distributions within the 
Table 1. Study Characteristics for Included Studies

\begin{tabular}{|c|c|c|c|c|c|c|c|c|c|c|c|}
\hline Article* & $\begin{array}{l}\text { Publication } \\
\text { Year }\end{array}$ & Country & $\begin{array}{l}\text { Specimen } \\
\text { Type }\end{array}$ & $\begin{array}{l}\text { Mean Age, } \\
\text { y (Range) }\end{array}$ & Specimen, $\mathrm{n}$ & Capsular Area & Study Modality & Staining Technique & $\begin{array}{l}\text { Tissue } \\
\text { Analysis }\end{array}$ & Nerves Identified & Disease \\
\hline $\begin{array}{c}\text { Rabinowicz and } \\
\text { Jacqueline }^{19}\end{array}$ & 1990 & Switzerland & Live humans & $61.3(30-90)$ & 52 & Unspecified & Histologic analysis & $\begin{array}{c}\text { Hematoxylin, } \\
\text { eosin, Gieson, } \\
\text { Luxol-fast-blue, } \\
\text { Loyez, Glees, } \\
\text { Tibor-pap, Gomori }\end{array}$ & Microscopic & Unspecified & $\begin{array}{c}\text { OA, AS, AVN, } \\
\text { FNFx }\end{array}$ \\
\hline Birnbaum et al. ${ }^{20}$ & 1997 & Germany & Cadaver & NS & 11 & Unspecified & Gross dissection & None & Macroscopic & $\begin{array}{l}\text { FN, ON, AON, } \\
\text { SGN, NQF, SN }\end{array}$ & Unspecified \\
\hline Gáspár et al. ${ }^{21}$ & 2004 & Hungary & Live humans & Adults, age NS & 22 & Unspecified & Immunohistochemistry & $\begin{array}{l}\text { Anti-NF, Anti-SP, } \\
\text { Anti-CGRP, } \\
\text { Anti-NKl }\end{array}$ & Microscopic & Unspecified & $\mathrm{OA}$ \\
\hline Kampa et al..$^{22}$ & 2007 & United Kingdom & Cadaver & $81(54-107)$ & 20 & Unspecified & Gross dissection & None & Macroscopic & $\begin{array}{l}\text { FN, ON, AON, } \\
\text { IGN, SGN, NQF, } \\
\text { SN }\end{array}$ & Unspecified \\
\hline Saxler et al. ${ }^{23}$ & 2007 & Germany & Live humans & $68.7(52-80)$ & 9 & Unspecified & Immunohistochemistry & $\begin{array}{l}\text { Anti-Sp, } \\
\text { Anti-CGRP }\end{array}$ & Microscopic & Unspecified & $\begin{array}{c}\text { FNFx, OA, } \\
\text { painless failed } \\
\text { THA }\end{array}$ \\
\hline Gerherdt et al. ${ }^{24}$ & 2012 & USA & Cadaver & $76.5(68-93)$ & 8 & $\begin{array}{l}\text { Anterior, posterior, } \\
\text { inferior, } \\
\text { superolateral }\end{array}$ & Histologic analysis & Gold chloride & Microscopic & Unspecified & $\mathrm{OA}$ \\
\hline Haversath et al. ${ }^{25}$ & 2013 & Germany & Live humans & $55.6(8-87)$ & 34 & Anterolateral & $\begin{array}{l}\text { Immunohistochemistry, } \\
\text { histologic analysis }\end{array}$ & $\begin{array}{c}\text { Anti-S100, } \\
\text { Anti-SP, Anti-NF, } \\
\text { Anti-nociceptin, } \\
\text { Anti-neuropeptide } \\
\text { Y }\end{array}$ & Microscopic & Unspecified & AVN, OA, FAI \\
\hline Desteli et al. ${ }^{26}$ & 2014 & Turkey & Live humans & $0.9(0.5-1.5)$ & 60 & Unspecified & Immunohistochemistry & Anti-S100 & Microscopic & Unspecified & Normal, DDH \\
\hline $\begin{array}{l}\text { Grzegorzewski } \\
\text { et al. } .^{27}\end{array}$ & 2014 & Poland & Live humans & $9.5(5-18)$ & 34 & Unspecified & Immunohistochemistry & Anti-S100, Anti-SP & Microscopic & Unspecified & $\mathrm{CP}, \mathrm{DDH}$ \\
\hline Short et al. ${ }^{28}$ & 2018 & Canada & Cadaver & $79.3 \pm 11.9$ & 13 & Anterior & Gross dissection, ultrasound & None & Macroscopic & FN, ON, AON & Unspecified \\
\hline
\end{tabular}

AON, accessory obturator nerve; AS, ankylosing spondylitis; AVN, avascular necrosis; CGRP, calcitonin gene-related peptide; CP, cerebral palsy; DDH, developmental dysplasia of the hip; FAI femoroacetabular impingement; FN, femoral nerve; FNFx, femoral neck fracture; IGN, inferior gluteal nerve; NF, neurofilament; NK1, neurokinin 1; NQF, nerve to quadratus femoris; NS, not specified; OA, osteoarthritis; ON, obturator nerve; SGN, superior gluteal nerve; SN, sciatic nerve; SP, substance P; THA, total hip arthroplasty.

*All included clinical studies were Level IV evidence. 


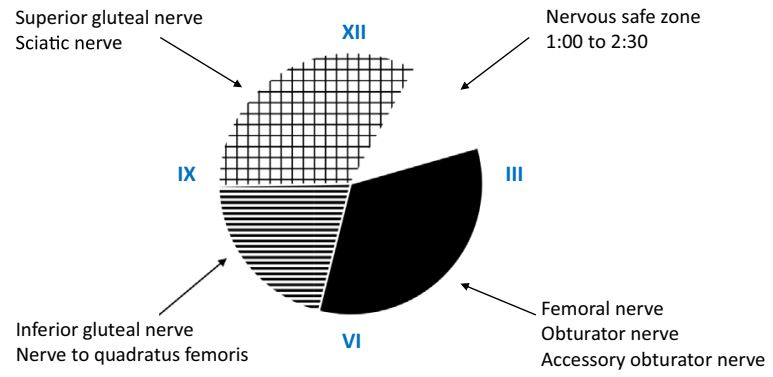

Fig 2. Right hip capsule innervation pattern.

hip capsule. ${ }^{24}$ This study demonstrated that the superolateral zone of the capsule had the greatest density of mechanoreceptors and sensory fibers followed by the anterior zone of the capsule. No sensory fibers were found in either the inferior or posterior zones of the hip capsule specimens.

A total of 94 pediatric patients were assessed by 2 in vivo studies. ${ }^{26,27}$ Thirty subjects had DDH. Both articles were level IV evidence. Both studies performed immunohistochemical analysis of hip capsule biopsies for neurogenic protein S-100 to demonstrate that pain and femoral head articular cartilage loss is associated with a greater expression of nerve fibers. They also demonstrated that patients with cerebral palsy had a significantly greater number of nerve fibers compared with patients with DDH. However, there were no statistically significant differences in nerve fiber expression between DDH and normal hips.

A total of 117 adult patients were assessed by 4 in vivo studies using light microscopy histology and immunohistochemistry. Eleven adults had FAI and 7 adults had dysplasia. The remaining subjects had OA, avascular necrosis (AVN), femoral neck fracture, or inflammatory arthritides. All 4 articles were level IV evidence. Two studies analyzed nerve fiber densities within the hip capsule among patients with OA. ${ }^{21,23}$ Saxler et al. ${ }^{23}$ found that patients with OA have significantly greater substance $\mathrm{P}$ and calcitonin gene-related peptide immunoreactive nerve fiber densities compared with hips without OA. However, Gáspár et al. ${ }^{21}$ found that this high nerve fiber density does not correlate with preand postoperative pain or disability. Another study performed a histologic analysis of nerve fibers among patients with OA, rheumatoid arthritis, ankylosing spondylitis, femoral neck fracture, and AVN. ${ }^{19}$ They demonstrated that in AVN, rheumatoid arthritis, and femoral neck fractures, the number of nerve fibers within the capsule decreases over time and are also transformed into fibrous tissue. They found that these transformed fibrous tissues do not transmit pain leading to less painful pathologies when compared with OA and ankylosing spondylitis, for which nerve fibers do not transform into fibrous tissues. Another study investigated capsule innervation among patients with AVN,
$\mathrm{OA}$, and FAI to find that there is a negative correlation with aging and pain fiber expression. ${ }^{25}$ They demonstrated that patients with AVN had a greater distribution of pain-associated nerve fibers in the superior aspect of the capsule but found no significant difference in nerve fiber distribution between patients with FAI and OA.

\section{Discussion}

The most important finding of this systematic review of 4 cadaveric and 6 in vivo studies was that normal and diseased hip capsule innervation is complex. Macroscopic analyses of cadaveric studies were able to demonstrate that a normal hip capsule is innervated by the sciatic and superior gluteal nerves posterosuperiorly, nerve to quadratus femoris and inferior gluteal nerve posteroinferiorly, and femoral and obturator nerves anteriorly. These studies also identified the existence of an internervous safe zone in the anterior-superior capsule, a common location where capsular incisions are made during hip-preservation surgery. Microscopic analyses of both cadaveric and in vivo studies were able to demonstrate that nerve fiber expression did not correlate with pain. This information can be used to guide hip-preservation surgery capsular management.

This investigation studied a complex subject regarding the unique innervation pattern of the hip capsule and its association with pain in hip pathology. Previous studies have demonstrated that joint pain is associated with increased activation of free nerve endings by inflammatory mediators such as prostaglandins, neuropeptides, bradykinin, and cytokines. ${ }^{29,30}$ However, there are limited number of studies that investigate the quantity and distribution pattern of nerve fibers in relation to pain. Among the studies included in this systematic review, Grzegorzewski et al. ${ }^{27}$ demonstrated a correlation between pain and the number of nerve fibers, specifically among pediatric patients with cerebral palsy. Saxler et al. ${ }^{23}$ found that patients with OA have significantly greater nerve fiber densities compared with hips without OA. However, Gáspár et al. ${ }^{21}$ found that this high nerve fiber density does not correlate with pre- and postoperative pain or disability. Rabinowicz and Jacqueline ${ }^{19}$ and Haversath et al. ${ }^{25}$ also found that aging is associated with decreased pain fiber expression and increased fibrous tissue formation. These studies demonstrate that pain seen among patients with hip pathology is multifactorial. Greater expression of nerve fibers more significantly affects pain level among pediatric patients. However, due to the negative correlation between age and nerve fiber density as well as fibrous tissue formation over time, adult patients are less affected by nerve fiber density. Instead, increased release of inflammatory mediators as previously described by Schaible et al. ${ }^{29}$ and Perrot and Guilbaud et al. ${ }^{30}$ and likely play the major role in pain among patients with $\mathrm{OA}$ and FAI. 
The studies analyzed in this review suggest that the hip capsule has a complex macroscopic and microscopic innervation that contributes to the overall hip pain found in hip pathologies. While several studies negate the role of nerve fiber quantity in relation to the amount of pain production, evidence from this review strongly suggest that higher nerve fiber expression does not correlate with painful hip pathology, particularly in adults. However, this systematic review was unable to determine the effects of nerve fiber manipulation on pain regardless of the number (or density) of nerve fibers. Nerve tension via stretch has been shown to cause pain, which is a likely contributing factor in the pain perceived due to joint effusion with capsular stretch. In addition to direct fiber transection from capsular incisions (interportal, T, H, or L capsulotomies), a separate similar mechanical stimulus may occur with capsular repair instrument penetration or suture strangulation. Capsular adhesions (e.g., capsulolabral scar, peripheral compartment adhesions to the femoral neck, or overlying musculotendinous units [iliopsoas, rectus femoris, gluteus minimus, iliocapsularis, piriformis]) also may induce a nerve stretch instigating pain. Capsular deficiency (and the subsequent femoral head instability) may similarly stimulate capsular nerve fibers, causing pain. Thus, it is clear that capsular management plays a role in pre- and postoperative pain.

\section{Limitations}

There are limitations to this review. First, the heterogeneity of the included studies including study subjects (i.e., cadaveric vs in vivo) and methods used to evaluate microscopic innervation (types of staining) limited direct comparisons of results. Second, the included clinical studies were primarily retrospective level IV evidence. In addition, we sought to investigate the hip capsule in hip-preservation surgery subjects. Unfortunately, there was limited evidence (1 study, 11 subjects) in FAI syndrome and dysplasia ( 1 study, 7 subjects) in adults. Further, it is possible that our stringent search protocol and limiters may have excluded other relevant studies on this topic, including those published in languages other than English.

\section{Conclusions}

The hip capsule has a complex macroscopic and microscopic innervation pattern with varying nerve fiber expression from at least 6 peripheral nerves. OA is associated with a greater expression of nerve fibers, although nerve fiber expression does not correlate with painful pathology.

\section{References}

1. Goddard NJ, Gosling PT. Intra-articular fluid pressure and pain in osteoarthritis of the hip. J Bone Joint Surg $\mathrm{Br}$ 1988;70:52-55.
2. Myers SR, Eijer H, Ganz R. Anterior femoroacetabular impingement after periacetabular osteotomy. Clin Orthop Relat Res 1999;363:93-99.

3. Wang $\mathrm{H}$, Zheng XF, Zhang $\mathrm{X}$, et al. Increasing substance $\mathrm{P}$ levels in serum and synovial tissues from patients with developmental dysplasia of the hip (DDH). ВMC Мusculoskelet Disord 2014;15:92.

4. Scott CEH, MacDonald DJ, Howie CR. 'Worse than death' and waiting for a joint arthroplasty. Bone Joint J 2019;1 01 B:941-950.

5. Frank JM, Harris JD, Erickson BJ, et al. Prevalence of femoroacetabular impingement imaging findings in asymptomatic volunteers: A systematic review. Arthroscopy 2015:31:1199-1204.

6. Gardner E. The innervation of the hip joint. Anat Rec 1948;101:353-371.

7. Lloyd-Roberts GC. Osteoarthritis of the hip; a study of the clinical pathology. J Bone Joint Surg Br 1955;37-B:8-47.

8. Biedert RM, Sanchis-Alfonso V. Sources of anterior knee pain. Clin Sports Med 2002;21:335-347.

9. Terjesen $\mathrm{T}$. The natural history of hip development in cerebral palsy. Dev Med Child Neurol 2012;54:951-957.

10. Meeus M, Nijs J. Central sensitization: A biopsychosocial explanation for chronic widespread pain in patients with fibromyalgia and chronic fatigue syndrome. Clin Rheumatol 2007;26:465-473.

11. Walters BL, Cooper JH, Rodriguez JA. New findings in hip capsular anatomy: Dimensions of capsular thickness and pericapsular contributions. Arthroscopy 2014;30:1235-1245.

12. Walters BL. Editorial Commentary: \#Capsule-ophilesRejoice! The evidence in support of diligent capsular management during hip arthroscopy can no longer be ignored. Arthroscopy 2020;36:124-126.

13. Abrams GD. Editorial Commentary: Not repairing the hip capsule after arthroscopy-What were we thinking? Arthroscopy 2018;34:319-320.

14. Moher D, Liberati A, Tetzlaff J, Altman DG, the PRISMA Group. Preferred reporting items for systematic reviews and meta-analyses: The PRISMA Statement. PLoS Med 2009;6:e1000097.

15. Howick J, Chalmers I, Glasziou P, et al. The 2011 Oxford CEBM Evidence Levels of Evidence (Introductory Document). Oxford Centre for Evidence-Based Medicine. http://www.cebm.net/index.aspx?ol/45653. Accessed April 26, 2020.

16. Harris JD, Brand JC, Cote MP, Dhawan A. Research Pearls: The significance of statistics and perils of pooling. Part 3: Pearls and pitfalls of meta-analyses and systematic reviews. Arthroscopy 2017;33:1594-1602.

17. Slim K, Nini E, Forestier D, Kwiatkowski F, Panis Y, Chipponi J. Methodological index for non-randomized studies (MINORS): Development and validation of a new instrument. ANZ J Surg 2003;73:712-716.

18. Slavin RE. Best evidence synthesis: An intelligent alternative to meta-analysis. J Clin Epidemiol 1995;48:9-18.

19. Rabinowicz T, Jacqueline F. Pathology of the capsular and synovial hip nerves in chronic hip diseases. Pathol Res Pract 1990;186:283-292.

20. Birnbaum K, Prescher A, Hessler S, Heller KD. The sensory innervation of the hip joint-an anatomical study. Surg Radiol Anat 1997;19:371-375. 
21. Gáspár L, Dezso B, Csernátony Z, et al. Capsular neuronal elements and their relation to pain reduction and functional improvement following total hip replacement. Int Orthop 2004;28:142-145.

22. Kampa RJ, Prasthofer A, Lawrence-Watt DJ, Pattison RM. The internervous safe zone for incision of the capsule of the hip. A cadaver study. J Bone Joint Surg Br 2007;89:97 1-976.

23. Saxler G, Löer F, Skumavc M, Pförtner J, Hanesch U. Localization of SP- and CGRP-immunopositive nerve fibers in the hip joint of patients with painful osteoarthritis and of patients with painless failed total hip arthroplasties. Eur J Pain 2007;1 1:67-74.

24. Gerhardt M, Johnson K, Atkinson R, et al. Characterisation and classification of the neural anatomy in the human hip joint. Hip Int 2012;22:75-81.

25. Haversath M, Hanke J, Landgraeber S, et al. The distribution of nociceptive innervation in the painful hip: A histological investigation. Bone Joint J 2013;95-B:770-776.
26. Desteli EE, Gülman AB, Imren Y, Kaymaz F. Comparison of mechanoreceptor quantities in hip joints of developmental dysplasia of the hip patients with normal hips. Hip Int 2014;24:44-48.

27. Grzegorzewski A, Jóźwiak M, Pawlak M, Modrzewski T, Buchcic P, Masłoń A. Hip joint pain in children with cerebral palsy and developmental dysplasia of the hip: Why are the differences so huge? BMC Musculoskelet Disord 2014;15:96.

28. Short AJ, Barnett JJG, Gofeld M, et al. Anatomic study of innervation of the anterior hip capsule: Implication for image-guided intervention. Reg Anesth Pain Med 2018;43: 186-192.

29. Schaible HG, Ebersberger A, Von Banchet GS. Mechanisms of pain in arthritis. Ann N Y Acad Sci 2002;966: 343-354.

30. Perrot S, Guilbaud G. Pathophysiology of joint pain. Rev Rhum Engl Ed 1996;63:485-492. 
Appendix Table 1. Search Strategy of Relevant Literature

- Search 1: (capsule[Title/Abstract] OR hip osteoarthritis[Title/Abstract] OR developmental dysplasia[Title/Abstract] OR femoral acetabular impingement[Title/Abstract]) AND (Hip[All Fields] AND innervation[All Fields]).

- Search 2: (capsule[Title/Abstract] OR hip osteoarthritis[Title/Abstract] OR developmental dysplasia[Title/Abstract] OR femoral acetabular impingement[Title/Abstract]) AND (hip osteoarthritis[All Fields] AND innervation[All Fields]).

- Search 3: (capsule[Title/Abstract] OR hip osteoarthritis[Title/Abstract] OR developmental dysplasia[Title/Abstract] OR femoral acetabular impingement[Title/Abstract]) AND (developmental dysplasia[All Fields] AND innervation[All Fields]).

- Search 4: (capsule[Title/Abstract] OR hip osteoarthritis[Title/Abstract] OR developmental dysplasia[Title/Abstract] OR femoral acetabular impingement[Title/Abstract]) AND (femoral acetabular impingement[All Fields] AND innervation[All Fields]). 